\title{
El tatuaje y su relación con características personales y sociales
}

\section{The tattoo and its relation with personal and social characteristics}

\author{
Alejandro D. Castro \\ Juan I. Aragonés \\ Universidad Complutense de Madrid
}

\section{Resumen}

Tatuarse es una práctica cada vez más frecuente en sociedades occidentales. Este trabajo analiza la relación existente entre el tatuaje y distintas variables de carácter personal y social. La muestra formada por 189 personas, de las cuales 65 eran hombres, cumplimentó un cuestionario compuesto, por un lado, por escalas correspondientes a los "cinco grandes": la necesidad de unicidad, la búsqueda de sensaciones, la autoestima, las actitudes hacia el tatuaje, y, por otro lado, por variables sociodemográficas y relativas al tatuaje. Los resultados muestran que las mujeres se tatúan más que los varones, que la realización de un tatuaje es más probable cuanto mayor es la presencia de otras personas tatuadas en el ámbito cotidiano, y que se tatúan más aquellos a quienes se atribuye una ideología más de izquierda. Además, los católicos se hacen un número significativamente menor de tatuajes que los ateos o agnósticos y hay una menor presencia de tatuados en el ámbito cotidiano cuanto más elevado es el nivel de estudios. Además, existe una relación significativa de la posesión de un tatuaje con mayores niveles en apertura a la experiencia, necesidad de unicidad, autoestima y actitudes hacia el tatuaje.

Palabras clave: tatuaje, diferencias individuales, rasgos de personalidad, variables sociodemográficas.

Nota del autor

Alejandro D. Castro, Facultad de Psicología, Departamento de Psicología Social, Universidad Complutense de Madrid; Juan I. Aragonés, Departamento de Psicología Social, Universidad Complutense de Madrid.

La correspondencia en relación con este artículo debe dirigirse a Juan I. Aragonés, Facultad de Psicología, Departamento de Psicología Social, Universidad Complutense de Madrid, Campus de Somosaguas s/n, C. P. 28223, Pozuelo de Alarcón (Madrid), España.

Dirección electrónica: jiaragones@psi.ucm.es 


\begin{abstract}
Tattooing is an increasingly frequent custom in western societies. This essay is an analysis of the existing connection between tattoos and several social and personal variables. The sample, comprising 189 people, of whom 65 were men, completed a questionnaire composed, firstly, of scales matching the Big Five personality factors, Need for Uniqueness, Sensation seeking, Self-esteem, Attitudes Toward Tattoos, and of sociodemographic and tattoo-related variables as well. The results show that women acquire tattoos more often than men, that the acquisition of a tattoo is more likely the more tattooed people are present in one's daily life, and that those adopting a rather left-wing ideology are more inclined to have a tattoo. Moreover, Catholics acquire a significantly lower number of tattoos than atheists or agnostics, and there is a smaller presence of tattooed people in a person's daily life the higher their educational level is. On the other hand, there is a significant association between having a tattoo and showing a higher score on Openness to experience, Need for Uniqueness, Self-esteem and Attitudes Toward Tattoos.
\end{abstract}

Keywords: tattoo, individual differences, personality traits, sociodemographic variables.

Una de las prácticas más comunes llevadas a cabo por la especie humana es la de modificar su aspecto externo con un determinado fin. En este sentido, cada vez es más frecuente en las sociedades occidentales que las personas decoren su cuerpo a través de métodos relativamente permanentes: body ornamentation u ornamentación corporal (Carmen, Guitar, \& Dillon, 2012). Bajo este concepto suelen englobarse tanto los tatuajes como las perforaciones (Wohlrab, Stahl, \& Kappeler, 2007a).

Esta investigación presta atención al tatuaje, ya que resulta una forma de decoración más llamativa que las perforaciones y sus implicaciones sociales son mayores. Además, su análisis resulta pertinente, en tanto es una práctica cada vez más presente en las sociedades occidentales ( Laumann \& Derick, 2006).
El tatuaje, proveniente del término tahitiano ta tau (Carmen et al., 2012), podría definirse como la inserción de un pigmento de color en la capa dérmica a través de una serie de perforaciones en la piel con el fin de crear una marca permanente (Tiggemann \& Hopkins, 2011). Esto origina que hacerse uno requiera, en la mayoría de los casos, de una toma de decisión reflexiva; los métodos que existen para eliminarlos son costosos y dolorosos o pueden dejar señales en la piel (Kosut, 2006).

A pesar de parecer un fenómeno reciente, cada vez con mayor presencia, el tatuaje posee una historia extensa, a través de la cual el significado, la importancia y las connotaciones dadas a dicha práctica hanido variando. Desde un punto de vista antropológico, existen evidencias de prácticas de modificación corporal que se remontan al Neolítico, como es el caso de la momia Ötzi, 
aunque no queda claro el significado que pudo asociarse a dichos diseños (Pabst et al., 2009). Por tanto, los orígenes del tatuaje moderno se sitúan en ciertas sociedades tribales (Carmen et al., 2012). En cuanto a su introducción en la cultura popular en occidente, ésta se produjo a finales del siglo xix, con el diseño de la primera máquina tatuadora. Así, a lo largo del siglo xx, los tatuajes empezaron a ganar en popularidad dentro de los militares entre la Primera y la Segunda Guerra Mundial como una forma de patriotismo simbólico. Posteriormente, durante la década de los sesenta el tatuaje se empezó a considerar como algo propio de subculturas, como la generación hippie (con una ideología de paz que se oponía a los militares), o los moteros, vinculados a tatuajes con una temática relacionada con movimientos antisociales. En la década de los setenta, los tatuajes fueron acaparados por otros movimientos, tales como la cultura punk, el movimiento gay y el feminismo, cuya práctica era una forma de reivindicar su libertad (DeMello, 2000).

Todos estos movimientos hicieron que poco a poco el tatuaje fuera introduciéndose en el imaginario de la población en general, hasta convertirse en una práctica muy habitual. La incorporación del tatuaje en el gusto se afianza cuando las figuras icónicas como cantantes, actores o deportistas llevan tatuajes, y los hacen deseables y atractivos para una buena parte de sus públicos (Kosut, 2006). Su elevada presencia es tal que estudios como el de Wohlrab et al. (2007b) apuntan que en sociedades occidentales
$10 \%$ de la población presenta algún tipo de modificación corporal, entre éstas se incluye el tatuaje. Pérez-Cotapos y Cossio (2006) afirman, por su parte, que entre $10 \%$ y $16 \%$ de los adolescentes y entre $3 \%$ y $8 \%$ de la población en general poseen algún tatuaje.

En definitiva, aunque existen estudios que muestran el aspecto negativo asociado con las personas tatuadas (Degelman \& Price, 2002; Seiter \& Hatch, 2005; Swami \& Furnham, 2007) Los tatuajes parecen haber trascendido cualquier barrera de la sociedad occidental, donde ha empezado a gozar de una gran aceptación. Personas de cualquier edad se tatúan (Deschesnes, Finès, \& Demers, 2006), a pesar de las restricciones legales de algunos lugares, donde se pide el consentimiento paterno para tatuar a menores de edad. El género tampoco es un impedimento, a pesar de que estudios como los de Armstrong et al. (2008) o Hawkes, Senn y Thorn (2004) han mostrado que existe una mayor estigmatización hacia las mujeres tatuadas. Lo que en un principio comenzó como algo propio de ciertos movimientos, como la generación hippie, las subculturas punk o motera, o el movimiento gay, ha empezado a emerger incluso como un símbolo de estatus en la cultura popular (Carmen et al., 2012), por igual entre hombres y mujeres, jóvenes y adultos.

Con todo lo visto hasta ahora, queda en evidencia la complejidad que rodea la práctica del tatuaje. Existe una gran cantidad de tipos de tatuajes con diseños específicos (Wohlrab, 
Stahl, Rammsayer, \& Kappeler, 2007b). No hay restricciones, se puede tatuar en cualquier zona del cuerpo humano, las repercusiones son diversas, algunas dependen de la facilidad para ocultar un tatuaje, debido a la percepción negativa qe proyectan las personas tatuadas en el espacio social (Degelman \& Price, 2002; Seiter \& Hatch, 2005; Swami \& Furnham, 2007). Las motivaciones y objetivos que llevan a una persona a hacerse un tatuaje en una zona descubierta no serán los mismos que para quienes prefieren tatuarse en áreas ocultas, prohibidas a la vista (Wohlrab et al., 2007a).

En definitiva, el tatuaje no consiste en un mero dibujo decorativo, grabado sobre la piel, su significado y sus repercusiones sobrepasan tal condición. Si bien es una práctica común, cuyo sentido social tiene un marco de referencia complejo no ha tenido resonancia en la psicología, en cuanto a estudios empíricos; el número de publicaciones en español es mínimo (Mejía, 2015). Los principales aspectos analizados desde esta disciplina han sido las motivaciones que llevan a las personas a tatuarse, los comportamientos asociados al tatuaje, la percepción social que se tiene de las personas tatuadas, o las diferencias existentes entre las personas en función de la posesión o carencia de un tatuaje.

\section{EI tatuaje en la psicología}

Por un lado, se han estudiado las principales motivaciones que impulsan a las personas a tomar la decisión de modificar su cuerpo a través de un tatuaje. Destaca la revisión llevada a cabo por Wohlrab y colaboradores (2007a), dentro de la cual pueden diferenciarse dos tipos de motivaciones: de carácter social y con una finalidad personal. De las motivaciones sociales puede destacarse la idea de embellecer el cuerpo o mostrar resistencia física al haber sido capaz de soportar el doloroso proceso (Wohlrab et al., 2007a), dotar al individuo de un sentido de pertenencia a un grupo determinado (Madrigal, 2005), desafiar las normas y el orden establecido (Delazar, 2005), o mostrar masculinidad y dominancia en varones, y fecundidad en las mujeres (Wohlrab et al., 2009). Desde un carácter más personal, el tatuaje puede dotar de cierta individualidad e influir en la creación y mantenimiento de la identidad (Tiggemann 6 Golder, 2006), aumentar la autoestima (Swami, 2011) expresar valores, ideas y sentimientos propios (Madrigal, 2005), representar la tradición cultural y espiritual propia (Wohlrab et al., 2007a), o satisfacer la adicción que puede generarse al tatuarse (Carmen et al., 2012).

La psicología ha puesto también su interés en analizar la posible relación existente entre el tatuaje y ciertos comportamientos. Estos trabajos se han centrado mayoritariamente en adolescentes, y relacionan la posesión del tatuaje con diferentes conductas desviadas. Braithwaite y colaboradores (2001), lo asocian con el consumo de alcohol, marihuana, antidepresivos y sedantes; Deschesnes, Finès y Demers (2006) con actividades ilegales, afiliación a pandillas, problemas con el juego y absentismo escolar, 
mientras Caroll, Riffenburgh, Roberts y Myhere (2002) con desórdenes alimenticios, actos violentos y suicidios.

Otro aspecto sobre el que la psicología ha mostrado interés es la percepción social que la población en general tiene de las personas tatuadas. Tal y como se apuntó con anterioridad, diversos estudios concluyen que existe una percepción social negativa hacia los tatuados. Las mujeres tatuadas son percibidas socialmente como, atractivas, motivadas, honestas, generosas, misteriosas, religiosas e inteligentes, aunque no tan atléticas (Degelman \& Price, 2002), y más promiscuas cuantos más tatuajes posean (Swami \& Furnham, 2007). Por otro lado, la credibilidad atribuida a una persona también es menor si ésta posee un tatuaje (Seiter \& Hatch, 2005).

Debido a la existencia de determinados comportamientos asociados al tatuaje, y a que la percepción social de los tatuados es versátil, cabe preguntarse en este punto qué clase de diferencias pueden existir entre tatuados y no tatuados. En primer lugar, se han llevado a cabo estudios con base en el análisis de la personalidad desde el modelo de los cinco grandes. Este modelo considera que la personalidad está estructurada en cinco rasgos o factores: Neuroticismo, Extraversión, Apertura a la experiencia, Responsabilidad y Amabilidad. En este campo se han obtenido resultados contradictorios, estudios como los llevados a cabo por Swami y compañeros (2012), así como Wohlrab y colaboradores (2007b), muestran que las personas tatuadas presentan mayores niveles deExtraversión, mientras que el de Tate y Shelton (2008) concluye que los tatuados puntúan más alto en Amabilidad y en Responsabilidad.

Hemos señalado la función del tatuaje en la configuración de identidad y de individualidad para quien los porta. La "Teoría de la unicidad", desarrollada por Snyder y Fromkin (1980), tendría cabida, entonces, a la hora de analizar por qué las personas se tatúan. Según esta teoría, el ser humano tiene la necesidad de ser semejante a los demás y a la vez necesita ser diferente y especial. Aquellos con mayores niveles de necesidad de unicidad buscarán, por tanto, objetos que les diferencien del resto, como puede ser un tatuaje. Distintos trabajos relacionados con este aspecto concluyen en efecto que las personas tatuadas muestran mayores niveles de necesidad de unicidad que los no tatuados (Swami et al., 2012; Tiggemann \& Hopkins, 2011; Tiggemann \& Golder, 2006).

El tatuaje también se ha relacionado con un mayor deseo de vivir experiencias y sensaciones que resulten novedosas (Swami et al., 2012; Stirn, Hinz, \& Brähler, 2006; Wohlrab et al., 2007b). Por otro lado, se ha observado que la autoestima aumenta en relación con la aceptación propia que expresó el individuo antes de hacerse el tatuaje (Swami, 2011), aunque al comparar a personas tatuadas con no tatuadas no se han obtenido diferencias significativas (Swami et al., 2012). Finalmente, se corroboró la asociación positiva entre poseer un tatuaje y 
presentar una actitud más favorable hacia dicha práctica (Swami et al., 2012).

Dada la falta de concordancia entre los resultados de algunas de las investigaciones, el objetivo de este trabajo es de carácter descriptivo, relaciona el tatuaje con características individuales y sociales. En concreto, se trata de conocer en qué medida el tatuaje está relacionado con variables de carácter sociodemográfico $\mathrm{y}$, por otro lado, determinar la relación entre ciertas variables disposicionales: personalidad, búsqueda de sensaciones y autoestima $\mathrm{y}$ psicosociales: necesidad de unicidad, actitudes hacia el tatuaje y presencia de tatuados en el ámbito cotidiano con el hecho de estar tatuado.

\section{Método}

\section{Participantes}

La muestra estaba compuesta por un total de 189 personas de nacionalidad española, de las cuales 124 eran mujeres y 65 eran hombres. La media de edad fue de 23.89 años (DT $=2.78$ ), dentro de un intervalo de 17 a 30 años. Del total de participantes, $7.3 \%$ tenían estudios inferiores a bachillerato, 23\% presentaba un nivel educativo de bachillerato y $68.6 \%$ restante tenían estudios universitarios o estaba cursándolos en este momento. Respecto a la ideología política, para cuya medición se utilizó una escala de 1 a 10 , donde 1 representaba la extrema izquierda y 10 la extrema derecha, se obtuvo una media de $4.27(\mathrm{DT}=1.52)$, es decir, una ligera tendencia a una ideología de izquierdas. En cuanto a la religión, 36.4\% de los participantes se declaró católico y $63.6 \%$ restante, ateo o agnóstico. Otra cuestión central fue la presencia constante de personas tatuadas en el ámbito cotidiano de los participantes, para lo cual se establecieron tres niveles: ninguna persona estaba tatuada en su ámbito cotidiano (14.3\%), solamente algunas estaban tatuadas $(15.7 \%)$ o la mayoría estaban tatuadas $(69.1 \%)$.

\section{Instrumentos}

Para alcanzar los objetivos propuestos, se elaboró un cuestionario compuesto por las variables sociodemográficas y cuestiones relacionadas con distintos aspectos del tatuaje, como la posesión o no de uno, el número de ellos, sus localizaciones, la edad a la que se hizo el primer tatuaje, la importancia dada al tatuaje, ya fuese un símbolo personal o algo superficial y a que pudiera ser exhibido (medidas a través de dos ítems con valores de 0 a 10), el nivel de satisfacción actual con los tatuajes (medido con una escala con intervalo de 1 a 100) y la presencia de personas tatuadas en sus ámbitos cotidianos.

En segundo término, se contempló una serie de escalas para medir las variables disposicionales y psicosociales. La primera de ellas fue la escala desarrollada por Terracciano y colaboradores (2005), utilizada para medir los factores de personalidad del Modelo de los Cinco Grandes. Dicha escala consta de 30 escalas bipolares de cinco puntos. Los valores de alfa de Cronbach 
obtenidos en este estudio para las cinco escalas fueron de 0.73 para la Responsabilidad, 0.71 para la Extraversión, 0.66 tanto para la Apertura a la experiencia como para la Responsabilidad, y 0.57 para el Neuroticismo.

Para medir la necesidad de unicidad o necesidad de diferenciarse de los demás, se llevó a cabo una adaptación en español de la Escala de Necesidad de Unicidad a partir de traducciones paralelas realizadas por los autores de la investigación. Esta escala, desarrollada por Snyder y Fromkin (1977), consta de 32 ítems a los que el participante debe mostrar su nivel de acuerdo a través de una escala tipo Likert de cinco puntos. Con la presente muestra se obtuvo un alfa de Cronbach de 0.83 .

La necesidad de buscar sensaciones y experiencias nuevas fue recogida a través de la "Forma V de la Escala de Búsqueda de Sensaciones" (EBS-V), elaborada por Zuckerman, Eysenck y Eysenck (1978), que consta de 40 ítems a los que se debe contestar de manera afirmativa o negativa. Para el presente estudio se utilizó la adaptación al español llevada a cabo por Pérez y Torrubia (1986). Con el fin de reducir el tamaño del cuestionario se acudió al análisis factorial llevado a cabo por Ferrando y Chico (2001), eliminando 24 ítems. Finalmente, para mejorar la fiabilidad hasta un nivel aceptable, se eliminaron tres ítems más, aquellos con menor correlación con el resto, se alcanzó un alfa de Cronbach de 0.70

La autoestima fue medida con base en la escala desarrollada por Rosenberg (1965), la cual está compuesta por diez ítems hacia los que el participante debe mostrar su acuerdo mediante una escala tipo Likert con los límites 1: Muy en desacuerdo y 5: Muy de acuerdo. En este caso, se recurrió a la adaptación al español elaborada por Martín-Albo, Núñez, Navarro y Grijalvo (2007). En el presente estudio se obtuvo un alfa de Cronbach de 0.85 .

Por último, las actitudes generales hacia el tatuaje se midieron aplicando la escala elaborada por Swami y colaboradores (2012), consistente en ocho pares de características opuestas relativas al tatuaje, entre cuyos extremos el participante debe posicionarse, a través de una escala de uno a siete. Por medio de un análisis factorial, la escala utilizada a efectos de análisis estaba compuesta por cuatro ítems, con los cuales se alcanzó una fiabilidad de 0.81 .

\section{Procedimiento}

El cuestionario se aplicó en línea a través de la plataforma SurveyMonkey. La muestra fue obtenida a partir de un muestreo no probabilístico mediante la técnica de bola de nieve. En primer lugar, los investigadores se contactaron con personas que tenían algún tatuaje. Éstos, tras cumplimentar el cuestionario, enviaron el enlace del cuestionario a otras personas que ellos conocían y que tenían tatuajes. Una vez alcanzado un número aceptable de participantes tatuados, se realizó el mismo procedimiento para obtener la submuestra de personas no tatuadas. El tiempo empleado por cada participante en 
cumplimentar el cuestionario era de quince minutos, aproximadamente.

\section{Análisis de datos}

En primer lugar, se estudió la relación entre los distintos aspectos del tatuaje y las variables sociodemográficas. Se consideró, también, la relación de la presencia de tatuados en el ámbito cotidiano con el resto de aspectos del tatuaje. Para estos análisis, se recurrió a las técnicas de contraste de $\chi^{2}$ y t de Student y a la correlación de Pearson según correspondía.

Posteriormente, se examinaron las diferencias entre tatuados y no tatuados en las distintas variables disposicionales y psicosociales. Debido a la existencia de ciertas variables que tenían relación con la posesión de un tatuaje, como fueron el género, la presencia de tatuajes en el ámbito cotidiano y la ideología política, se llevó a cabo por un lado un ANOVA 2 (tatuaje vs no tatuaje) x 2 (varón vs mujer), y por otro lado un ANOVA 2 (tatuaje vs no tatuaje) x 3 (ninguna persona lleva tatuaje vs algunas lo llevan vs la mayoría lo lleva), considerando, en ambos casos, la ideología política como covariable.

\section{Resultados}

Del total de entrevistados, - $\mathrm{N}=189$-, un $47.08 \%$ estaba tatuado. En una primera descripción de éstos se observa, en primer lugar que la edad media a la que se hicieron el primer tatuaje era de 19.19 años $(\mathrm{DT}=3.09)$, que $37.08 \%$ de los tatuados llevaba un solo tatuaje, la mayor frecuencia, y que el número medio de tatuajes que llevaban en el cuerpo era de 2.32 $(\mathrm{DT}=1.39)$. El nivel de satisfacción mostrado hacia sus propios tatuajes, medido a través de una escala de 1 a 100 , obtuvo un nivel medio de 93.73 (DT = 10.85). Al utilizar la escala de 0 a 10 para medir la importancia dada por los participantes a que el tatuaje fuera un elemento con valor personal y significativo, se obtuvo una media de $8.96(\mathrm{DT}=3.17)$. En cuanto a la importancia dada por los participantes a que el tatuaje fuera algo visible para los demás, se obtuvo un valor medio de $3.79(\mathrm{DT}=3.51)$, con base en una escala de 0 a 10. En lo referente a la localización de los tatuajes, $17.8 \%$ de ellos fue hecho en la parte superior de la espalda; $13.1 \%$, en el antebrazo; $11.5 \%$, en el brazo; $11 \%$, en el abdomen; $10.5 \%$, en la parte inferior de la pierna; $7.9 \%$, en el pie; $7.3 \%$, en los hombros; $6.8 \%$, en el pecho; $3.7 \%$, en la parte baja de la espalda; $3.7 \%$, en las manos; $3.7 \%$, en la parte superior de la pierna; $2.1 \%$, en la garganta y el $0.5 \%$, en la cara.

A continuación, se analizó en qué medida diversos aspectos del tatuaje tienen relación con distintas variables sociodemográficas (tabla 1). Se encontraron diferencias significativas al relacionar la posesión de un tatuaje con el sexo $\left(\chi^{2}=4.11 ; \mathrm{gl}=1 ; p<0.05\right)$, la presencia de tatuados en el ámbito cotidiano $\left(\chi^{2}=9.98 ; \mathrm{gl}=\right.$ $2 ; p<0.01)$ y la ideología política $(\mathrm{t}=-2.02$; $\mathrm{gl}$ $=187 ; p<0.05)$. Así, los tatuajes son realizados en mayor medida por mujeres, su realización es más probable cuanto mayor es la presencia de 
otras personas tatuadas en el ámbito cotidiano, y están relacionados con una ideología más de izquierda. En cuanto al número de tatuajes, se encontró relación con la religión de los participantes $\left(\chi^{2}=6.56 ; \mathrm{gl}=2 ; p<0.05\right)$, de manera que los católicos se hacen un número significativamente menor tatuajes, por lo común tan solo uno, mientras que los ateos muestran una mayor predisposición a hacerse más de dos.

Al analizar la presencia de tatuados en el ámbito cotidiano, se observó que guarda relación con el sexo $\left(\chi^{2}=9.41 ; \mathrm{gl}=2 ; p<0.01\right)$ y el nivel de estudios $\left(\chi^{2}=14.74 ; \mathrm{gl}=4 ; p<0.01\right)$. Así, existe una mayor presencia de personas tatuadas en el ámbito cotidiano de las mujeres, y una menor presencia de tatuados cuanto más elevados son los estudios de los participantes. Por otro lado, aunque no se obtuvieron diferencias significativas a la hora de comparar la presencia de tatuajes en el ámbito cotidiano con el número de tatuajes $\left(\chi^{2}=8.54 ; \mathrm{gl}=4 ; p=\right.$ $0.07)$ y con la ideología política $(\mathrm{F}=2.871 ; \mathrm{gl}=$ $2 ; p=0.06)$, sus niveles de significación fueron muy cercanos a 0.05 . Lo anterior evidencia que cuanto mayor fue la presencia de tatuados en el ámbito cotidiano mayor fue el número de tatuajes que llevaban los participantes, con una tendencia, además, hacia una ideología de izquierda.

Tabla 1

Porcentajes y valores medios de las variables sociodemográficas según distintos aspectos relacionados con el tatuaje

\begin{tabular}{|c|c|c|c|c|c|c|c|c|c|c|}
\hline & \multicolumn{2}{|c|}{ Tatuaje } & \multicolumn{3}{|c|}{ Número de tatuajes } & \multicolumn{3}{|c|}{ Tatuaje en el ámbito cotidiano } & \multicolumn{2}{|c|}{$\begin{array}{c}\text { Edad primer } \\
\text { tatuaje }\end{array}$} \\
\hline & Sí & No & 1 & 2 & +2 & Ninguno & Algunos & La mayoría & $M$ & $D T$ \\
\hline \multicolumn{11}{|l|}{ Género } \\
\hline Varón & $36,9^{*}$ & 63,1 & 33,3 & 37,5 & 29,2 & $24,6^{\star \star}$ & 64,6 & 10,8 & 19,96 & 3,46 \\
\hline Mujer & 52,4 & 47,6 & 38,5 & 23,1 & 38,5 & 8,9 & 72,6 & 18,5 & 18,91 & 2,91 \\
\hline \multicolumn{11}{|l|}{ Nivel de estudios } \\
\hline Básicos & 71,4 & 28,6 & 10,0 & 50,0 & 40,0 & $21,4^{\star \star}$ & 50,0 & 28,6 & 17,30 & 2,41 \\
\hline Bachillerato & 38,6 & 61,4 & 41,2 & 35,3 & 23,5 & 27,3 & 52,3 & 20,5 & 19,71 & 3,92 \\
\hline Universitarios & 47,3 & 52,7 & 40,3 & 21,0 & 38,7 & 9,2 & 77,9 & 13,0 & 19,36 & 2,86 \\
\hline \multicolumn{11}{|l|}{ Religión } \\
\hline Católico & 44,1 & 55,9 & $53,3^{*}$ & 26,7 & 20,0 & 14,7 & 73,5 & 11,8 & 19,07 & 3,45 \\
\hline Ateo-agnóstico & 47,9 & 52,1 & 28,1 & 28,1 & 43,9 & 14,3 & 67,2 & 18,5 & 19,19 & 2,93 \\
\hline \multicolumn{11}{|l|}{ Ámbito cotidiano } \\
\hline Ninguno & $22,2^{\star \star \star}$ & 77,8 & 50,0 & 33,3 & 16,7 & & & & 19,83 & 5,08 \\
\hline Algunos & 48,5 & 51,5 & 43,8 & 25,0 & 31,2 & & & & 19,51 & 3,01 \\
\hline La mayoría & 63,3 & 36,7 & 10,5 & 31,6 & 57,9 & & & & 17,95 & 2,34 \\
\hline \multicolumn{11}{|l|}{ Ideología } \\
\hline$M$ & $4,03^{*}$ & 4,48 & 4,27 & 3,92 & 3,87 & 4,89 & 4,20 & 4,00 & & \\
\hline$D T$ & 1,39 & 1,61 & 1,30 & 0,93 & 1,74 & 1,42 & 1,52 & 1,55 & & \\
\hline
\end{tabular}


Más tarde, se examinaron las diferencias entre los tatuados y los no tatuados en las distintas variables disposicionales y psicosociales (ver tablas 2 y 3). En primer lugar, existe un efecto significativo de la posesión de un tatuaje sobre la necesidad de unicidad, la autoestima y la actitud hacia el tatuaje. En ambos análisis de varianza, los tatuados obtuvieron puntuaciones más altas que los no tatuados en la escala de necesidad de unicidad $(\mathrm{F} 1=4.11 ; \mathrm{F} 2=3.46$; $\mathrm{gl}=1 ; p<0.05)^{1} \mathrm{y}$ un nivel significativamente mayor de autoestima $(\mathrm{F} 1=4.31 ; \mathrm{F} 2=5.38$; gl $=1 ; p<0.05)$. Los tatuados obtuvieron además una puntuación menor que los no tatuados en la escala de actitudes hacia el tatuaje, con una evidente actitud más positiva hacia ellos $(\mathrm{F} 1=$ 88.65; F2 = 40.41; $\mathrm{gl}=1 ; p<.001)$.

En cuanto a las diferencias en los cinco grandes, respecto al factor Apertura a la experiencia se obtuvieron valores de significación diferentes en ambos análisis de varianza. Por ello en esta variable se recurrió a t de Student, de ahí que se obtuvo una relación significativa entre la posesión de un tatuaje y la Apertura a la experiencia, por lo tanto, los tatuados puntuaron más alto que los no tatuados $(\mathrm{t}=3.515 ; \mathrm{gl}=187 ; p<0.01)$.

Tabla 2

Estadísticos descriptivos y resultados de las comparaciones entre la posesión de un tatuaje y el género (2x2) para las distintas variables dependientes

\begin{tabular}{|c|c|c|c|c|c|c|c|c|c|c|c|c|c|c|c|c|}
\hline \multirow[t]{2}{*}{ Variable } & \multicolumn{2}{|c|}{ No tatuado } & \multicolumn{2}{|c|}{ Tatuado } & \multicolumn{2}{|c|}{ Mujer } & \multicolumn{2}{|c|}{ Varón } & \multicolumn{2}{|c|}{ Test de varianza } & \multicolumn{2}{|c|}{ Efecto del tatuaje } & \multicolumn{2}{|c|}{ Efecto género } & \multicolumn{2}{|c|}{ Interacción } \\
\hline & $M$ & $S D$ & $M$ & $S D$ & $M$ & $S D$ & $M$ & $S D$ & $F$ & $R^{2}$ & $F_{1}$ & $\eta^{2}$ & $F$ & $\eta^{2}$ & $F$ & $\eta^{2}$ \\
\hline Neuroticismo & 2,54 & 0,65 & 2,39 & 0,61 & 2,55 & 0,62 & 2,31 & 0,63 & $2,58^{*}$ & 0,05 & 3,05 & 0,02 & $6,92^{* *}$ & 0,04 & 0,18 & $<0,01$ \\
\hline Extraversión & 3,61 & 0,69 & 3,79 & 0,60 & 3,78 & 0,61 & 3,54 & 0,71 & 2,25 & 0,05 & 2,44 & 0,01 & $4,26^{*}$ & 0,02 & 0,12 & $<0,01$ \\
\hline Ape. experiencia & 3,51 & 0,64 & 3,84 & 0,63 & 3,74 & 0,65 & 3,53 & 0,65 & $6,81^{\text {***}}$ & 0,13 & $8,72^{\star \star}$ & 0,04 & 2,17 & 0,01 & 0,75 & $<0,01$ \\
\hline Responsabilidad & 3,68 & 0,64 & 3,60 & 0,66 & 3,70 & 0,64 & 3,53 & 0,65 & 1,10 & 0,02 & 1,25 & $<0,01$ & 3,41 & 0,02 & 0,30 & $<0,01$ \\
\hline Amabilidad & 3,79 & 0,49 & 3,78 & 0,63 & 3,88 & 0,55 & 3,60 & 0,53 & $3,23^{*}$ & 0,07 & 0,62 & $<0,01$ & $12,09^{\star \star}$ & 0,06 & 0,18 & $<0,01$ \\
\hline Nec. unicidad & 3,20 & 0,44 & 3,35 & 0,38 & 3,26 & 0,43 & 3,30 & 0,39 & $2,96^{*}$ & 0,06 & $4,11^{*}$ & 0,02 & 1,12 & $<0,01$ & 0,01 & $<0,01$ \\
\hline Búsq. sensac. & 0,48 & 0,23 & 0,53 & 0,20 & 0,50 & 0,21 & 0,50 & 0,23 & 1,48 & 0,03 & 2,50 & 0,01 & 0,15 & $<0,01$ & 0,80 & $<0,01$ \\
\hline Autoestima & 3,50 & 0,49 & 3,64 & 0,50 & 3,49 & 0,50 & 3,71 & 0,46 & $3,90^{* *}$ & 0,08 & $4,31^{*}$ & 0,02 & $10,31^{\star \star}$ & 0,05 & 0,43 & $<0,01$ \\
\hline Actitudes tatuaje & 3,66 & 1,31 & 2,05 & 0,90 & 2,72 & 1,35 & 3,26 & 1,40 & $29,54^{\star \star \star}$ & 0,39 & $88,65^{\star \star *}$ & 0,32 & 1,96 & 0,01 & $4,91^{*}$ & 0,03 \\
\hline
\end{tabular}

${ }^{\star} p<0,05 .{ }^{*} p<0,01 .{ }^{*} p<0,001$

$1 \mathrm{~F}_{1}$ hace referencia al valor del efecto del tatuaje obtenido en el ANOVA 2 (tatuaje $v s$ no tatuaje) $\mathrm{x} 2$ (varón $v s$ mujer), mientras que $\mathrm{F}_{2}$ se refiere al obtenido en el ANOVA 2 (tatuaje $v s$ no tatuaje) $\times 3$ (ninguna persona lleva tatuaje $v s$ algunas lo llevan vs la mayoría lo lleva). 
Tabla 3

Estadísticos descriptivos y resultados de las comparaciones entre la posesión del tatuaje y la presencia de tatuados en el ámbito cotidiano (2x3) para las distintas variables dependientes

\begin{tabular}{|c|c|c|c|c|c|c|c|c|c|c|c|c|c|c|}
\hline \multirow[t]{2}{*}{ Variable } & \multicolumn{2}{|c|}{ Ninguno } & \multicolumn{2}{|c|}{ Algunos } & \multicolumn{2}{|c|}{ La mayoría } & \multicolumn{2}{|c|}{ Test de varianza } & \multicolumn{2}{|c|}{ Efecto del tatuaje } & \multicolumn{2}{|c|}{ Efecto del ámbito } & \multicolumn{2}{|c|}{ Interacción } \\
\hline & $M$ & $S D$ & $M$ & $S D$ & $M$ & $S D$ & $F$ & $R^{2}$ & $F_{2}$ & $\eta^{2}$ & $F$ & $\eta^{2}$ & $F$ & $\eta^{2}$ \\
\hline Neuroticismo & 2,75 & 0,61 & 2,43 & 0,62 & 2,39 & 0,67 & 1,35 & 0,04 & 0,89 & $<0,01$ & 1,74 & 0,02 & 0,06 & $<0,01$ \\
\hline Extraversión & 3,13 & 0,77 & 3,79 & 0,56 & 3,80 & 0,67 & $6,80^{* \star *}$ & 0,18 & 3,16 & 0,02 & $4,03^{*}$ & 0,04 & $0,73^{* *}$ & 0,06 \\
\hline Ape. experiencia & 3,01 & 0,62 & 3,78 & 0,59 & 3,77 & 0,61 & $9,24^{\star \star \star}$ & 0,23 & 0,98 & $<0,01$ & $11,28^{\star \star \star \star}$ & 0,11 & 0,61 & $<0,01$ \\
\hline Responsabilidad & 3,59 & 0,79 & 3,63 & 0,58 & 3,74 & 0,78 & 1,76 & 0,05 & 0,03 & $<0,01$ & 1,13 & 0,01 & $4,04^{\star}$ & 0,04 \\
\hline Amabilidad & 3,60 & 0,64 & 3,80 & 0,56 & 3,87 & 0,43 & 0,78 & 0,02 & 0,02 & $<0,01$ & 1,08 & 0,01 & 0,14 & $<0,01$ \\
\hline Nec. unicidad & 3,06 & 0,56 & 3,31 & 0,38 & 3,29 & 0,40 & $2,96^{\star \star}$ & 0,09 & $3,46^{*}$ & 0,02 & 1,00 & 0,01 & 0,80 & $<0,01$ \\
\hline Búsq. sensac. & 0,40 & 0,24 & 0,52 & 0,21 & 0,55 & 0,22 & $2,62^{*}$ & 0,08 & 0,05 & $<0,01$ & 1,58 & 0,02 & 2,72 & 0,03 \\
\hline Autoestima & 3,44 & 0,52 & 3,58 & 0,50 & 3,58 & 0,44 & 1,47 & 0,05 & $5,38^{*}$ & 0,03 & 0,01 & $<0,01$ & 2,04 & 0,02 \\
\hline Actitudes tatuaje & 4,14 & 1,41 & 2,77 & 1,28 & 2,38 & 1,23 & $21,89^{\star \star \star}$ & 0,42 & $40,41^{\star \star \star}$ & 0,18 & $5,93^{\star \star}$ & 0,06 & 0,10 & $<0,01$ \\
\hline
\end{tabular}

Además de la relación de la posesión de un tatuaje con algunas de las variables disposicionales y psicosociales, se obtuvieron efectos significativos del sexo y el ámbito cotidiano sobre algunas de esas variables. Se observó que el sexo tiene relación con tres de los cinco factores de la personalidad, como el Neuroticismo, la Extraversión y la Amabilidad, y con el Autoestima (ver tabla 2). Así, las mujeres mostraron niveles mayores en Neuroticismo ( $\mathrm{F}$ $=6.92 ; \mathrm{gl}=1 ; p<0.01)$, Extraversión $(\mathrm{F}=4.26$; $\mathrm{gl}=1 ; p<0.05)$ y Amabilidad $(\mathrm{F}=12.09 ; \mathrm{gl}=1$; $p<0.01$ ), mientras que los hombres obtuvieron puntuaciones más altas que las mujeres en Autoestima $(\mathrm{F}=10.31 ; \mathrm{gl}=1 ; p<0.01)$. Por otro lado, se observó un efecto significativo de la interacción entre el tatuaje y el sexo en la variable relativa a las actitudes hacia el tatuaje $(\mathrm{F}=4.91 ; \mathrm{gl}=1 ; p<0.05)$, aunque con un tamaño del efecto mínimo en comparación al tamaño del efecto del tatuaje sobre dicha variable dependiente.

En cuanto al efecto de la presencia de tatuados en el ámbito cotidiano sobre las distintas variables, se observó la existencia de relaciones entre los distintos niveles de dicha variable con la Extraversión, la Apertura a la experiencia y la Actitud hacia el tatuaje (ver tabla 3). En primer lugar, se observa que cuanto mayor es la presencia de tatuajes en el ámbito cotidiano de los participantes mayores son los niveles de Extraversión $(\mathrm{F}=4.03 ; \mathrm{gl}=2$; $p<0.05)$ y de Apertura a la experiencia, $(\mathrm{F}=$ 11.28; $\mathrm{gl}=2 ; p<0.001)$. Por último, cuanto más favorable es la actitud de los participantes hacia el tatuaje, mayor es el número de tatuados 
presente en sus ámbitos cotidianos. $(\mathrm{F}=5.93 ; \mathrm{gl}$ $=2 ; p<0.01)$. En este caso se obtuvo, además, un efecto significativo de la interacción entre el tatuaje y el ámbito cotidiano en las dimensiones de Extraversión $(\mathrm{F}=5.73 ; \mathrm{gl}=2 ; p<0.01)$, $\mathrm{y}$ de Responsabilidad ( $\mathrm{F}=4.04 ; \mathrm{gl}=2 ; p<0.05)$.

\section{Discusión}

El tatuaje, sin duda, es un fenómeno que tiene cada día mayor presencia en la sociedad occidental. Lo mismo ocurre en el contexto español, donde se ha convertido en una práctica bastante generalizada, sobre todo teniendo en cuenta estudios como el de Sigma Dos, el cual apunta que $26 \%$ de los jóvenes lleva al menos un tatuaje ("La revolución del tatuaje", 2012). Sin embargo, esta gran prevalencia no se ha visto correspondida desde la psicología en cuanto al número de estudios empíricos llevados a cabo (Mejía, 2015). Aquellos que se han realizado se centran principalmente en analizar los motivos que llevan a las personas a tatuarse, los comportamientos asociados al tatuaje, la percepción social que se tiene de los tatuados y las diferencias existentes en ciertas características individuales en función de estar o no tatuado.

En este estudio se concluye que la posesión de un tatuaje se relaciona con el sexo, de manera que entre las personas entrevistadas había más mujeres tatuadas que no tatuadas, mientras que esto no se producía en el caso de los hombres. Este resultado merece destacarse en la medida que los estudios muestran una mayor estigmatización hacia las mujeres tatuadas que hacia los hombres (Armstrong et al., 2008; Hawkes, Sonn, \& Thurn, 2004); a este respecto no existen estudios en la sociedad española. No obstante, se observa que el tatuaje resulta en la actualidad una práctica muy normalizada $\mathrm{y}$, por tanto, cabe pensar que sea aceptada por la mayor parte de la población. En segundo lugar, el tatuaje se relacionó con una ideología más de izquierda. La religión tuvo también relación con el tatuaje, de manera que los ateos y agnósticos se hacen un mayor número de tatuajes con respecto a los católicos. Por otro lado, se observó que la presencia de tatuados en el ámbito cotidiano de las personas es mayor entre los que llevaban un tatuaje, es decir, se ejerce influencia social sobre los pares a la hora de hacerse un tatuaje. Este resultado se encuentra en la línea junto con los señalados por Madrigal (2005) y Wohlrab y colaboradores (2007a), quienes ponían en evidencia el papel del tatuaje como símbolo identitario de pertenencia a un grupo. Se podría concluir, entonces, que es más probable entre los jóvenes tatuados que éstos sean ideológicamente progresistas, sin vínculos religiosos y así convivan con pares también tatuados.

En la segunda parte se han estudiado las relaciones entre estar o no tatuado y distintas variables disposicionales y situacionales. En lo referente a los rasgos de personalidad, según el Modelo de los Cinco Grandes, los tatuados puntuaron de manera significativamente mayor 
en la Apertura a la experiencia. Este resultado abunda en la discrepancia ya existente sobre cuáles son los rasgos de la personalidad que se relacionan con el tatuaje, ya que en algunos trabajos se muestra que los tatuados puntuaban más alto en Extraversión (Swami et al., 2012; Wohlrab et al., 2007b), mientras que en otro lo hacían en Amabilidad y Responsabilidad (Tate \& Shelton, 2008). El conjunto de estos resultados lleva a considerar que estos factores no se relacionan con el tatuaje de una forma generalizable. Esa variabilidad de resultados puede deberse a cuestiones relacionadas con las muestras que se han utilizado en cada caso, así como a otras de carácter transcultural, en el sentido de que unos $u$ otros contextos pueden facilitar que un tipo determinado de personas se tatúen.

En este momento conviene recordar la falta de concordancia obtenida en esta investigación entre los dos análisis de varianza con la variable Apertura a la experiencia frente al resto de variables. La discrepancia con el estadístico $\mathrm{F}$ se ve afectado por el diferente número de comparaciones entre variables que conlleva cada tipo de análisis de varianza, este efecto puede ser la causa de tal discrepancia, por ello se realizó el análisis con la t de Student.

En lo referente a la necesidad de unicidad, los resultados obtenidos corroboran los hallados en investigaciones previas (Swami et al., 2012; Tiggemann \& Hopkins, 2011; Tiggemann \& Golder, 2006). Los tatuados tienen, por tanto, una mayor necesidad de ser distintivos $y$ especiales. El tatuaje sirve entonces como un elemento permanente que les diferencia del resto y que les confiere identidad e individualidad. La importancia dada por los individuos a ser diferente puede relacionarse a su vez con teorías evolucionistas como la de Carmen y colaboradores (2012), para quienes tatuarse el cuerpo puede suponer una forma de perpetuar los genes, en tanto que llevar un tatuaje puede llamar la atención de los individuos del sexo contrario.

En cuanto a la autoestima, se observa que ésta tiene una relación significativa con el tatuaje, de manera que los tatuados tienen una autoestima mayor, al contrario de lo que concluyeron Swami y colaboradores (2012). Sin embargo, el propio Swami (2011) acentúa el papel del tatuaje a la hora de mejorar la autoestima, mientras que llevar alguno aumenta la percepción de unicidad y se destaque en una sociedad donde la imagen se torna como un elemento fundamental. El tatuaje podría tener además una relación con una mejora en la percepción de la imagen corporal, se puede asemejar a operaciones de cirugía plástica, tal y como ilustran Ballén y Castillo (2015). Empero, los autores recomiendan realizar investigaciones que permitan confirmar esta relación. Todos estos resultados ponen de manifiesto la necesidad de estudiar más en profundidad el papel de la unicidad, la autoestima y la imagen corporal, pero no sólo en cuanto a las relaciones lineales entre variables, sino tratando también de fusionarlas para entender mejor el proceso de toma de decisión de tatuarse. 
En conclusión, en este trabajo se ha puesto en evidencia la relación que ciertas variables personales y situacionales tienen con el tatuaje, observándose la importancia de la apertura a la experiencia, la necesidad de unicidad, la autoestima y la actitud hacia el tatuaje, además del sexo, el nivel de estudios, la ideología política, la religión y la red social a la hora de explicar este fenómeno. No obstante, en algunos casos los resultados obtenidos se encuentran en contradicción con otras investigaciones. Esto puede deberse, entre otros motivos, a que se consideraron todos los tatuajes por igual, sin considerar sus localizaciones, su visibilidad ni la motivación que llevó a tatuarse. El tener en cuenta estos aspectos en estudios futuros permitirá conocer con mayor precisión el papel personal y comunicacional que tiene el tatuaje.

Entre las limitaciones de esta investigación pueden destacarse el pequeño número de participantes, así como la diferencia entre hombres y mujeres; además de la baja fiabilidad de algunas de las escalas utilizadas. Ambas cuestiones pueden ser abordadas en investigaciones ulteriores, si se aumenta la muestra y mejora el diseño de las escalas con menor fiabilidad. Aunque de cara al futuro, bien pueden plantearse tres campos de interés que van más allá del carácter descriptivo en el que se encuadra la presente investigación. En primer lugar, sería interesante prestar atención al proceso de toma de decisión de tatuarse, tal y como se ha señalado anteriormente. Un segundo aspecto estaría relacionado con el análisis de la percepción de los tatuados sobre su propio cuerpo y sus tatuajes y cuál es la importancia del tatuaje a la hora de construir y modificar la identidad del tatuado. Y, en un tercer nivel, sería relevante estudiar la influencia que ejerce el contexto social, especialmente los pares, a la hora de tatuarse.

\section{Referencias}

Armstrong, M., Roberts, A., Koch, J., Saunders, J., Owen, D., \& Anderson, R. (2008). Motivation for contemporary tattoo removal. Archives of Dermatology, 144, 879-884.

Ballén, J. E., \& Castillo, J.A. (2015). La práctica del tatuaje y la imagen corporal. Revista Iberoamericana de Psicología: Ciencia y Tecnología, 8, 103-109.

Braithwaite, R., Robillard, A., Woodring, T., Stephens, T., \& Arriola, K. J. (2001). Tattooing and body piercing among adolescent detainess: Relationship to alcohol and other drug use. Journal of Substance Abuse, 13, 5-16.

Carmen, R. A., Guitar, A. E., \& Dillon, H. M. (2012). Ultimate Answers to Proximate Questions: The Evolutionary Motivations Behing Tattoos and Body Piercings in Popular Culture. Review of General Psychology, 16, 134-143.

Carroll, S. T., Riffenburgh, R. H., Roberts, T. A., \& Myhre, E. B. (2002). Tattoos and body piercings as indicators of adolescent risk-taking behaviors. Pediatrics, 109(6), 1021-1027. 
Degelman, D., \& Price, N. D. (2002). Tattoos and ratings of personal characteristics. Psychological Reports, 90, 507-514.

Delazar, M. E. (2005). The relationship between self-esteem, objectified body consciousness, personality traits and body modification: An exploratory study (Tesis doctoral). Pennsylvania, USA: Indiana University.

DeMello, M. (2000). Bodies of inscription: A cultural history of the modern tattoo community. Durham, NC: Duke University Press.

Deschesnes, M., Finès, P., \& Demers, S. (2006). Are tattooing and body piercing indicators of risk-taking behaviours among high school students? Journal of Adolescence, 29, 379393.

Ferrando, P., \& Chico, E. (2001). The construct of sensation seeking as measured by Zuckerman's SSS-V and Arnett's AISS: a structural equation model. Personality and Individual Differences, 31, 1121-1133.

Hawkes, D., Senn, C., \& Thorn, C. (2004). Factors that influence attitudes toward women with tattoos. Sex Roles, 50, 593-604.

Kosut, M. (2006). An Ironic Fad the Commodification and Consumption of Tattoos. The Journal of Popular Culture, 39, 1035-1048.

La revolución del tatuaje (2012, 1 mayo). Publico. Recuperado de http://www.publico. es/culturas/revolucion-del-tatuaje.html

Laumann, A. E., \& Derick, A. J. (2006). Tattoos and body piercings in the United States: A national data set. Journal of the American Academy of Dermatology, 55, 413-421.

Madrigal, M. A. (2005). Representación Social del Tatuaje en Jóvenes Tatuados entre 18 y 25 Años de Edad (Tesis de licenciatura). San Pedro: Universidad Fidélitas.

Martín-Albo, J., Núñez, J. L., Navarro, J. G., \& Grijalvo, F. (2007). The Rosenberg SelfEsteem Scale: Translation and Validation in University Students. The Spanish Journal of Psychology, 10, 458-467.

Mejía, P. (2015). La Psicología y el Tatuaje: una aproximación bibliométrica (Trabajo Fin de Grado). España: Universidad Complutense de Madrid,

Pabst, M. A., Letofsky-Papst, I., Bock, E., Moser, M., Dorfer, L., Egarter-Vigl, E., \& Hofer, F. (2009). The tattoos of the Tyrolean Iceman: a light microscopial, ultrastructural and element analytical study. Journal of Archaeological Science, 36, 2335-2341.

Pérez, J., \& Torrubia, R. (1986). Fiabilidad y validez de la versión española de la Escala de Búsqueda de Sensaciones (Forma V). Revista latinoamericana de Psicología, 18, 7-22.

Pérez-Cotapos, M. L., \& Cossio, M. L. (2006). Tatuajes y perforaciones en adolescentes. Revista Médica de Chile, 134, 1322-1329.

Rosenberg, M. (1965). Society and the adolescent self-image. Princeton, NJ: Princeton University Press.

Seiter, J. S., \& Hatch, S. (2005). Effect of tattoos on perceptions of credibility and attractiveness. Psychological Reports, 96, 1113-1120. 
Snyder, C. R., \& Fromkin, H. L. (1977). Abnormality as a positive characteristic: The development and validation of a scale measuring need for uniqueness. Journal of Abnormal Psychology, 86, 518-527.

Snyder, C. R., \& Fromkin, H. L. (1980). Uniqueness: The human pursuit of difference. Nueva York: Plenum Press.

Stirn, A., Hinz, A., \& Brähler, E. (2006). Prevalence of tattooing and body piercing in Germany and perception of health, mental disorders, and sensation seeking among tattooed and body-pierced individuals. Journal of Psychosomatic Research, 60, 531534.

Swami, V. (2011). Marked for life? A prospective study of tattoos on appearance anxiety and dissatisfaction, perceptions of uniqueness, and self-esteem. Body Image, 8, 237-244.

Swami, V., \& Furnham, A. (2007). Unattractive, promiscuous and heavy drinkers: Perceptions of women with tattoos. Body Image, 4, 343352.

Swami, V., Pietschnig, J., Bertl, B., Nader, I. W., Stieger, S., \& Voracek, M. (2012). Personality Differences between Tattooed and non-Tattooed Individuals. Mental \& Physical Health, 111, 97-106.

Tate, J. C., \& Shelton, B. L. (2008). Personality correlates of tattooing and body piercing in a college sample: The kids are alright. Personality and Individual Differences, 45, 281-285.
Terracciano et al. (2005). National Character Not Reflect Mean Personality Trait Levels in 49 Cultures. Science, 310, 96-100.

Tiggemann, M., \& Golder, F. (2006). Tattooing: An expression of uniqueness in the appearance domain. Body Image, 3, 309-315. Tiggemann, M., \& Hopkins, L. A. (2011). Tattoos and piercings: Bodily expressions of uniqueness? Body Image, 8, 245-250.

Wohlrab, S., Stahl, J., \& Kappeler, P. M. (2007a). Modifying the body: Motivations for getting tattooed and pierced. Body Image, 4, 87-95 Wohlrab, S., Stahl, J., Rammsayer, T., \& Kappeler, P. M. (2007b). Differences in Personality Characteristics Between BodyModified and Non-Modified Individuals: Associations With Individual Personality Traits and Their Possible Evolutionary Implications. European Journal of Personality, 21, 931-951.

Wohlrab, S., Fink, B., Kappeler, P. M., \& Brewer, G. (2009). Perception of human body modification. Personality and Individual Differences, 46, 202-206.

Zuckerman, M., Eysenck, S. B., Eysenck, H. (1978). Sensation seeking in England and America: cross-cultural, age, and sex comparisons. Journal of Consulting and Clinical Psychology, 46, 139-149.

Recibido el 6 de junio de 2016

Revisado el 18 de agosto de 2016

Aceptado el 15 de septiembre de 2016 